Aus der chirurg. Klinik in Lyon (Dir. Prof. Dr. A. Poncet).

\title{
Klinische, pathogenetische und therapeutische Studie über die gastrischen Krisen bei der Tabes dorsalis.
}

Von Dr. A. Cade, Privatdozent für innere Medizin und Dr. R. Leriche, Privatdozent für Chirurgie in Lyon.

Unter den visceralen Krisen bei der Tabes nehmen die gastrischen Krisen unbestreitbar den ersten Platz ein, woraus sich ihre Häufigkeit und Bedeutung erklärt. Man beobachtet sie in 30 Proz. der Fälle von vorgeschrittener lokomotorischer Ataxie (Röstel). Gewöhnlich treten sie bei ein und demselben Kranken wiederholt auf und lenken die Aufmerksamkeit des Arztes auf sich durch ihre für gewöhnlich alarmierenden, mitunter sogaridramatisch anmutenden klinischen Erscheinungsweisen. Zuerst wurde ihr Wesen von Duchenne (Boulogne) verkannt. Erst 1866 brachte sie Delamarre in seiner unter Raymonds Leitung verfaßten Dissertation in Zusammenhang mit der Tabes dorsalis. Späterhin waren es namentlich Topinard im Jahre I 869 und vor allem Charcot, der teils in seinen „Leçons“, teils in den Arbeiten seiner Schüler in klassischer Weise die klinische Physiognomie dieser Visceralgien festlegte. Seitdem sind zahlreiche Arbeiten über diesen Gegenstand erschienen, und namentlich in den letzten Jahren hat die einschlägige Literatur eine wertvolle Bereicherung erfahren. Ganz allmählich vollzog sich ein hochinteressanter Umschwung in unseren pathogenetischen Anschauungen, welcher naturgemä $B$ den Arzt dahin führen mußte, wenigstens bei gewissen Formen von tabischen Gastralgien die Hilfe des Chirurgen in Anspruch zu nehmen.

An der Hand der wichtigsten hierüber erschienenen Arbeiten über diese Frage und hauptsächlich auf Grund der neuesten Forschungsergebnisse beabsichtigen wir in dem vorliegenden 
Vortrage ein getreues klinisches Bild der tabischen gastrischen Krisen zu zeichnen und die pathogenetische Auffassung, auf dic man sich dabei zu stützen hat, zu erörtern. Nachdem wir dann näher auf die Grundzüge der internen Behandlung eingegangen sind, werden wir uns besonders mit den chirurgischen Eingriffen, die gegen die hartnäckigen und sich wiederholenden Formen der tabischen gastrischen Krisen vorgeschlagen worden sind, zu beschäftigen haben, indem wir die Indikationen, Technik und die Resultate der betreffenden Operationsmethoden besprechen.

I.

Klinische Studie. (Dr. Cade.)

Wir werden in diesem Abschnitt nacheinander die Symptome der gastrischen Krise, und zwar zunächst des gewöhnlichen Typus, dann die atypischen Formen, ihren Verlauf, das Verhalten der Verdauungsfunktionen während des anfallsfreien Intervalles, ihre Beziehungen zu der allgemeinen Symptomatologie der Tabes und schließlich die wichtigsten diagnostischen Punkte zu behandeln haben.

A. Beschreibung der gewöhnlichen Form.

Die wesentlichsten Charakterzüge der gastrischen Krise im Verlaufe der lokomotorischen Ataxie sind seit langem den Ärzten geläufig.

Die Schmerzen, das Erbrechen und die Veränderungen des Allgemeinzustandes stellen im wesentlichen die hauptsächlichsten Kardinalsymptome des klinischen Krankheitsbildes dar. Sie treten oftmals nach einem kurzen Prodomalstadium auf oder äußern sich als unbestimmtes Mißbehagen, allgemeiner Schwächezustand usw.

Die Schmerzen treten im linken Epigastrium und Hypochondrium auf und strahlen in das ganze Abdomen, in den Rücken, in die Lendengegend und um den Thorax herum aus. Sie gehen oft mit einem sehr unangenehmen Gefühl des Eingeengtseins (sog. Gürtelgefühl) einher.

Sie beginnen in brüsker Weise und erreichen rasch ihren Höhepunkt. Sie sind in der Regel sehr heftig, manchmal sogar 
unerträglich, lanzinierend oder stechend, sie verursachen den Kranken große Qualen und zwingen sie oft, die mannigfaltigsten und bizarresten Haltungen einzunehmen. Auf jeden Fall sug. gerieren sie ihnen mannigfaltige Vergleiche und Ausdrücke.

Das Erbrechen stellt das zweite Kardinalsymptom der Krise dar. Es tritt während des Verdauungsgeschäftes oder auch im nüchternen Zustande auf und hat je nach den Umständen das Erbrechen oder Nichterbrechen von mehr oder weniger reichlichen und veränderten Speiseresten zur Folge. Dann erfolgt stets unter erheblichen Anstrengungen wiederholtes Herauspressen von schleimiger Flüssigkeit, die meist zähe, seltener flüssig, wässerig und oft mit Galle gefärbt ist. Das Erbrechen wiederholt sich mehrmals, ist unstillbar und kann bei manchen Patienten die Entleerung einer großen Flüssigkeitsmenge zur Folge haben. Einige produzieren schließlich nur geringe Quantitäten von Speichel und Schleim, dann bleiben ihre Anstrengungen machtlos. Es ist dies das Stadium des „trockenen Erbrechens" (Fournier), das gewiß die schmerzhafteste Variante darstellt.

Nicht selten sind die entleerten Mengen mit einigen Blutspuren vermengt. Die eigentlichen Hämatemesen werden hingegen nur selten festgestellt. Sie geben der Krise, die sie komplizieren, ein ganz besonderes klinisches Gepräge, die uns instand setzt, sie unter die Formen der tabischen Gastralgie einzureihen und zu beschreiben.

Während des Erbrechens pflegen einzelne Singultus oder Eruktationen oft die Phase relativer Ruhe zu stören, in welcher sich der Kranke befindet.

Eine hochgradige Beeinträchtigung des Allgemeinbefindens begleitet ständig den Schmerz und das Erbrechen. Der Patient ist mehr oder minder stark deprimiert, und seine Niedergeschlagenheit zeigt alle Grade, von der einfachen Indolenz angefangen bis zum Stupor. Das Gesicht ist bleich, die Gesichtszüge verzogen, einen ängstlichen Ausdruck verratend, die Augen tief eingesunken, manchmal starr oder gleichgültig, manchmal in einer Lageveränderung eine Verminderung der Schmerzen suchend. So präsentiert sich dem Kliniker der unglückliche Tabiker, der das Opfer einer „Magenkolik“" ist. Sein Puls ist gespannt, ge- 
wöhnlich beschleunigt (ca. Ioo-i2o Schläge pro Minute), die Herzgeräusche sind oft verstärkt. Die Verminderung der Pulsschläge stellt, wie bereits $\mathrm{Charcot}$ richtig beobachtet hat, die Ausnahme dar. Dieses Verhalten steht im Gegensatz zu zahlreichen anderen visceralen Krisen. Der Blutdruck ist sehr häufig erhöht (Pal, Heitz und Noréro) und schwankt zwischen i 8 und $22 \mathrm{ccm} \mathrm{Hg}$. Der Patient hat häufig Gefühle von Schwindel und Ohnmachten. Die Pupillen sind gewöhnlich dilatiert, was besonders bemerkenswert erscheint hinsichtlich der für gewöhnlich zu beobachtenden Myosis der Tabiker. Die Temperatur bleibt normal und sinkt sogar zuweilen bis unter die Norm. Der Durst ist lebhaft. Die Harnmengen sind spärlich und arm an Chloraten. Oft tritt sogar eine Anurie an Stelle der Oligurie. v. Noorden hat auf die häufige Anwesenheit von Aceton und Acetessigsäure im Urin hingewiesen, was den Ärzten kaum überraschend erscheinen dürfte, denen die Häufigkeit der Ketonkörper in den Urinen von Patienten mit Inanition oder Erkrankungen des Verdauungstraktus bekannt ist.

Um dieses klinische Krankheitsbild zu vervollständigen, müssen wir noch die Darmerscheinungen schildern, die sich zu den funktionellen Magenbeschwerden hinzugesellen und Aufschlüsse resümieren, welche die Untersuchung des Abdomens und die Resultate des Magenchemismus uns liefern.

Die Untersuchung des Abdomens ergibt in der Regel eine Abflachung desselben, während die Auftreibung die Ausnahme darstellt. Die Bauchwand ist kontrahiert, und die Versuche zu palpieren erhöhen die reflektorische Wachsamkeit der Musculi recti („défense musculaire“). Die parietale Sensibilität im Bereiche des Epigastriums ist häufig gestört und manchmal reicht die Hyperästhesie bis zu der Iateralen Thoraxwand im Bereich der letzten Interkostalräume, besonders auf der linken Seite. Umgekehrt hat man ebenso Anästhesie in demselben Gebiet im Verlauf der gastrischen Krise feststellen können (Heit z und Lortat-Jacob).

Die Veränderungen der tiefen Sensibilität sind nicht immer denjenigen der oberflächlichen Sensibilität entsprechend, und recht oft findet man neben einer Hyperästhesie der Haut das Fehlen des schmerzhaften epigastrischen Druckpunktes und sogar 
Hypästhie des Plexus solaris, wie J.C.R oux ganz richtig beobachtet hat.

Die Beteiligung des Darmes an dem klinischen Krankheitsbild der tabischen Gastralgie ist konstant, wenn auch sehr variabel. Man notiert in allen Fällen Obstipation. Jedoch kann diese Obstipation bei manchen Patienten einen außergewöhnlichen Grad erreichen, mitunter sogar mit einer Verhaltung der Winde einhergehen. Bei anderen Patienten wird man, jedoch viel seltener, im Gegenteil Durchfälle, und zwar manchmal sogar sehr reichliche und profuse Diarrhöen konstatieren mit peristaltischen Darmgeräuschen und mehr oder minder häufigem Abgang von Winden, und es ist interessant, die Koexistenz oder Aufeinanderfolge der Entorrhöe und der Gastrorrhöe zu konstatieren. Manchmal kann sich die sekretorische Flutwelle am Eingang des Verdauungstraktus unter dem Bilde einer mehr oder minder reichlichen Sialorrhöe bemerkbar machen. Die Darmerscheinungen können im Verlaufe der "Magenkolik" ziemlich ausgesprochen sein, so daß man alsdann den beobachteten Symptomenkomplex als das Resultat der Kombination derselben mit einer echten intestinalen Krise bezeichnen kann im Hinblick darauf, daß die Koexistenz oder Aufeinanderfolge der beiden genannten Visceralgien keineswegs selten $z$ beobachten ist ( $\mathrm{Vulpian})$. Es ist sogar berechtigt, mit Loeper zu sagen, daB eine gewisse Anzahl von Krisen, die als gastrische angesprochen werden, eher als intestinale aufzufassen sind, weil bei ihnen die Darmerscheinungen vorherrschen: funktionelle Störungen, schmerzhafte Lokalisationen, äußerliche Veränderungen des Abdomens (Auftreibung und viel häufiger Einziehung), Sitz der oberflächlichen Hyperästhesie: all diese Symptome lenken die Aufmerksamkeit mehr auf den Darmkanal als auf den Magen hin.

Über die Ergebnisse des Magenchemismus im Verlaufe der tabischen Gastralgie können wir uns kurz fassen. Es ist heutzutage sehr bezeichnend, daß sich keine bestimmte Regel darüber aufstellen läßt.

Die Verschiedenheiten des Chemismus von einem Patienten zum anderen und die Möglichkeit von Schwankungen im Verlaufe von ein und derselben Krise sind ganz bekannte Dinge. Ursprünglich wurde der Hyperchlorhydrie (Sahli, Rosenthal, 
A. Robin usw.) ein Platz angewiesen, der ihr jedoch nach dem Ergebnis späterer Untersuchungen zu Unrecht zukommt, da die Hypopepsie in solchen Fällen häufiger zu beobachten ist als die Hyperpepsie. Die echte Hypersekretion mit Hyperchlorhydrie (intermittierender Magensaftfluß) ist seltener. Zusammengefaßt resümiert die folgende Formel: ,jeder Patient macht seine Krise nach der Beschaffenheit seiner Magenschleimhaut durch" am besten die allgemeine Ansicht über diesen Gegenstand ( $\mathrm{H}$ a yem und Babon, A. Mathieu).

B. Atypische Formen.

Wir haben soeben an die hauptsächlichsten Merkmale der klassischen Form der tabischen Gastralgie erinnert. Es bleibt uns übrig, ihre atypischen Formen zu besprechen, die zum größten Teile von Charcot und A.Fournier ausführlich geschildert worden sind. Sie können nach mehreren Gesichtspunkten rubriziert werden.

Die Anomalie kann zunächst aus dem sehr verschiedenen Grad der Intensität der hauptsächlichsten Komponenten des Symptomenkomplexes hervorgehen. Bald sehr schwache, bald hingegen außerordentlich heftige Symptome können der Krise ein etwas eigenartiges Gepräge verleihen. Dies würde jedoch die Individualisierung wirklicher klinischer Formen keineswegs rechtfertigen und auf jeden Fall keine ausführliche Beschreibung erfordern.

Anders steht es hingegen mit der Verwischung einzelner Hauptsymptome mit entsprechendem Hervortreten eines von ihnen, welche die wirklichen „frusten" Formen der tabischen Magenkrise schaffen. Wir heben als solche hervor:

I. den reinen gastralgischen Typus, wo die sehr lebhaften Schmerzen nicht mit Erbrechen einhergehen (Fournier),

2. den vomitären Typus, wo das Erbrechen den Hauptbestandteil der Krise unter Ausschluß der Schmerzen darstellt (Fournier, R. Lépine),

3. den eiskalten Typus,

wo die Kollapserscheinungen (Abkühlung, Cyanose usw.) einen hohen Grad erreichen und an Cholera erinnern können, um 
so mehr, als reichliche Durchfälle in solchen Fällen nicht zu den Seltenheiten gehören.

Bei einer gewissen Zahl von Patienten ist das Atypische darin gegeben, daß für gewöhnlich akzessorische Symptome das Krankheitsbild beherrschen oder besser noch ungewöhnliche Symptome im Verlaufe der typischen Krise auftreten. Bei der mit Flatulenz einhergehenden Form sind die Schmerzen wenig ausgesprochen. Das Erbrechen ist oft genug durch einfachen Brechreiz ersetzt. Häufig sind Eruktationen. Wiederholte peristaltische Geräusche passieren den Darm und führen zum Austritt zahlreicher Winde durch den Anus. Der Magen und sogar der Darm zeigen manchmal einen deutlichen Meteorismus. All die genannten Symptome sind der Ausdruck einer echten aërophagischen Krise. Diese Form kommt übrigens nur bei neuropathischen Tabikern zur Beobachtung.

Das Auftreten einer Magen blutung, auf das zuerst Charcot und Vulpian und in neuester Zeit Rauzier unsere Aufmerksamkeit gelenkt haben, ist bei der „Magenkolik“ der Tabiker selten. Sie tritt für gewöhnlich am Ende der intensiven und wiederholten Krisen auf. Sie äußert sich in Gestalt von Bluterbrechen, das wenig reichhaltig ist und manchmal von einer deutlichen Melaena gefolgt sein kann. Das durch das Erbrechen zutage geförderte Blut ist fast immer schwarz, und die auf diese Weise komplizierte Krise trägt somit zu Recht die von Charcot gewählte Bezeichnung ,schwarze Krise“. Die im Verlaufe der tabischen Gastralgie zu beobachtende Magenblutung kann die Folge einer früheren Läsion der Magenschleimhaut (hauptsächlich Ulcus, manchmal Gastritis oder Neubildung) sein. Froment und Tolot haben diese Komplikation besonders häufig in den Fällen gefunden, wo die späteren Befunde bei der Autopsie das gleichzeitige Vorhandensein eines organischen Magenleidens ergeben haben. In anderen Fällen erklärt die Ruptur eines kleinen Magengefäßes unter dem Einfluß des erhöhten Blutdruckes oder infolge heftigen Erbrechens den blutigen Charakter der Krise (Neumann, Tedesko). Die Präexistenz sklerotischer Läsionen der Magenarterien begünstigt augenscheinlich das Auftreten von Blutungen. Endlich existieren Beobachtungen, in denen die sorgfältigste Untersuchung der Magenschleimhaut auch nicht die ge- 
ringsten Anhaltspunkte für die Entstehungsursache der Blutungen gegeben hat (Mathieu, Kollarits). Man sieht sich dazu veranlaßt, diffuse kongestive Erscheinungen und vasomotorische Störungen dafür verantwortlich zu machen, die sicherlich einige Analogie mit den Ekchymosen der Haut aufzuweisen haben, die StrauB im Verlaufe der lanzinierenden Schmerzen ausführlich beschrieben hat.

Wir müssen in diesem Zusammenhange noch einiger atypischer Formen der Magenkrisen gedenken, zunächst der mit Bulimie einhergehenden Form, die sehr selten zu beobachten ist, wo die Kombination des pathologischen Appetites mit schmerzhaften Erscheinungen das klinische Krankheitsbild charakterisiert, und endlich der Form, die den Reichmannschen Symptomenkomplex vortäuscht oder besser ausgedrückt, des intermittierenden Typus dieses Symptomenkomplexes. Die letztere Abart hat Soupault vortrefflich geschildert, der selbst einen Fall dieser Art beobachtet hat und einen ähnlichen Fall in der Arbeit von Reichmann fand. Diese Krise ist charakterisiert durch die fortwährende Absonderung eines an Salzsäure überreichen Magensaftes. Wir werden später auf die übrigen unterscheidenden Merkmale dieser besonderen Form der gastrischen Krise hinweisen. Das gleichzeitige Bestehen derselben mit einer Magenerkrankung (funktionelle Dyspepsie oder organisches Magenleiden) führt im allgemeinen zu Modifikationen des klinischen Krankheitsbildes, ebenso wie die Kombination mit anderen tabischen Krisen. Wir werden bald die einen wie die anderen kennen lernen, wollen aber jetzt schon die allgemeinen Charakterzüge des Verlaufes der gastrischen Krise hervorheben: die Verschiedenheiten derselben, ihre anormale, entweder zu kurze oder zu lange Dauer, können zur Schilderung von anormalen Verlaufsformen führen, auf welche näher einzugehen, uns die folgenden Ausführungen entheben.

\section{Verlauf.}

Die typischen gastrischen Krisen beginnen brüsk, am häufigsten ohne Prodomalerscheinungen, und endigen ebenfalls brüsk, indem der Patient ohne Übergang aus einem Zustand von fast absoluter Intoleranz des Magens in einen solchen von normaler 
Funktion seines Verdauungsapparates übergeht. Bald sind die Krisen kurz, von ein- bis zweitägiger, manchmal auch bloß stündlicher Dauer, manchmal sind sie lang, können I4 Tage bis drei Wochen und darüber dauern. Es handelt sich dabei nicht um das anormale Andauern ein und derselben Krise, sondern um eine Serie von gastralgischen Paroxysmen, gewissermaßen um eine Art „état de mal" mit fieberhaften Krisen. Die tabische Visceralgie wiederholt sich mehr oder weniger häufig, in größeren oder kürzeren Intervallen. Die Krisen zeigen im allgemeinen die Tendenz, sich zeitlich zu nähern und eine längere Dauer anzunehmen. Man begreift leicht, wie unerträglich ein derartiger $\mathrm{Zu}$ stand für den armen Tabiker werden kann und welche Gefahren daraus resultieren: ungenügende Nahrungsaufnahme, Anämie, Niedergeschlagenheit und oft Morphinismus. Der Tod kann das Resultat dieser zunehmenden Verschlechterung des Allgemeinbefindens sein. Er kann ferner, übrigens in nicht seltenen Fällen, im Verlauf der Krise selbst im Coma oder unter Kollapserscheinungen eintreten (Vulpian). Bei dieser letzteren Möglichkeit sind ziemlich oft profuse Diarrhöen zu beobachten.

Bei anderen, sicher zahlreicheren Tabikern ziehen sich die Krisen in die Länge, nehmen an Zahl und Intensität ab, um schließlich ganz zu verschwinden.

Die Koexistenz eines organischen oder funktionellen Magenleidens bringt interessante Veränderungen im Verlaufe wie im klinischen Krankheitsbilde dieser Paroxysmen selbst mit sich, auf die wir im folgenden Abschnitt zu sprechen kommen.

D. Interparoxystisches Stadium.

Bei den gewöhnlichen Formen der gastrischen Krisen ist der anfallsfreie Intervall durch keinerlei Magenstörungen gekennzeichnet. Es ist dem aber nicht immer so. Die tabische Visceralgie kann auch mit einem organischen oder funktionellen Magenleiden einhergehen, welche sie, abgesehen von ihren eignen Äußerungen durch den heftigeren und paroxystischeren Charakter beeinflußt, den sie den Symptomen des Magenleidens oft aufdrückt. Dieses seinerseits wiederum beeinflußt die Visceralgie in folgender Weise: Die Krisen beginnen und endigen im allgemeinen weniger brüsk; in ihrem Intervall bleiben übrigens 
variable Verdauungsstörungen bestehen; Diätfehler, Aufregungen, Überarbeitung, Menstruation können zu neuen Krisen führen, während hingegen eine antidyspeptische Diät imstande ist, sie in die Länge zu ziehen. Es sind dies in der Tat Einflüsse, die sich bei den reinen Formen der tabischen Gastralgie nicht spüren lassen.

Die Koexistenz derselben mit dem Ulcus oder Carcinoma ventriculi muß wohl bekannt sein, da sie oft große diagnostische Schwierigkeiten bereitet.

Die Vergesellschaftung mit der Gastritis ist noch viel gewöhnlicher (Hayem), mag es sich um eine syphilitische Gastritis, oder noch viel öfter um eine alkoholische Gastritis, oder schließlich um medikamentöse Gastritis handeln (bedingt durch Jodide, Arsenik oder Quecksilber). Bei Morphiumsüchtigen beobachtet man ebenfalls, wie Mathieu und J.C.Roux, Bauer und Dobrovici treffend hervorgehoben haben, gewisse Modifikationen des Symptomenkomplexes der gastrischen Krisen, die wochenlang andauern können mit übrigens weniger ausgesprochenen Erscheinungen als während der freien Paroxysmen. Es handelt sich dann zumeist um neuropathische Individuen.

Die Neuropathie ist imstande, eine Veränderung im Verlaufe der Krise herbeizuführen und kann ihr oftmals Erscheinungen hinzufügen, die ihr Mit-im-Spiel-sein enthüllen: Schreie, Träume, extreme Aufregung, normale Diurese oder sogar Polyurie an Stelle der gewöhnlichen Oligurie. Sie kann sogar im Intervall der der Tabes eigentümlichen Paroxysmen rein neuropathische Magenkrisen hervorrufen, die unter dem Einflusse geeigneter Behandlung, speziell bei Isolierung und Psychotherapie zurückgehen.

Ganz kürzlich hat A. Mathieu die Aufmerksamkeit auf kolikartige Krisen gelenkt, die im Intervall der Paroxysmen der ausgesprochenen tabischen Gastralgie auftreten und imstande sind, eine anormale Fortdauer derselben zu bewirken. Diese kolikartigen Krisen sind kurz, wiederholen sich aber mehr oder weniger häufig. Sie ziehen eine geringere gastrische Intoleranz nach sich, oft nur ein- oder zweimaliges Erbrechen am Tage und, was sehr wichtig ist, sie erscheinen stets gleichzeitig mit den Koliken und gehen in den heftigsten Fällen gleichzeitig mit 
schweren Diarrhöen einher. Der letzte Stuhlgang markiert dann das Ende der Krise.

E. Beziehungen der gastrischen Krisen zu den übrigen Symptomen der Tabes.

Die gastrischen Krisen treten selten zum ersten Male in einem vorgeschrittenen Stadium der Tabes auf. Sie machen sich am liebsten im Initialstadium der Erkrankung bemerkbar und stellen sogar ziemlich häufig das Initialsymptom derselben dar, Von 224 Tabesfällen sah A, Fournier die Erkrankung mit Magendarmerscheinungen beginnen. Diese können sich sogar lange Zeit (Io Jahre in einem Falle von Ewald, 20 Jahre in einem Falle von Touche) vor jedem anderen Symptom der Sklerose der Hinterstränge äußern. Man begreift in solchen Fällen alle diagnostischen Schwierigkeiten und die mögliche $\mathrm{Zu}$ gehörigkeit der bei der intermittierenden Form der Reich mannschen Krankheit zu beobachtenden Symptomenkomplexe oder schließlich deren Rubrizierung unter die Reihe der essentiell gastrischen Krise (Debove) oder des periodischen Erbrechens (v. Leyden), dessen Existenz zu so lebhaften Diskussionen geführt hat.

Es ist jedoch immerhin selten, daß man - wenigstens längere Zeit hindurch - kein anderes Symptom der Tabes feststellen kann: Erloschensein der Patellarsehnenreflexe oder wenigstens der Achillessehnenreflexe, Myosis, Strabismen und mehr oder weniger vorübergehende Diplopie, beginnende Inkoordination, mehr oder wenig ausgesprochenes Rombergsches Phänomen, Blasen-, Mastdarmstörungen und namentlich lanzinierende Schmerzen. Letztere können parallel mit der gastralgischen Krise verlaufen, oder, wie es zumeist der Fall ist, mit ihr alternieren, ihr entweder vorangehen oder sich ihr anschließen.

Einige Symptome der Tabes können ausnahmsweise intermittieren, so z. B. das Westphalsche und Argyll-Robertsonsche Phänomen, jedoch treten sie bei jeder Magenkrise wieder in Erscheinung und sind dadurch imstande, die Diagnose sicher zu stellen (Heitz und Lortat-Jacob, Mantoux).

Die Vergesellschaftung oder das Alternieren der Gastralgie mit anderen tabischen Krisen ist ziemlich gewöhnlich. Wir haben 
bereits auf die Beziehungen zu der Enteralgie und die ziemlich häufige Existenz gemischter gastro-intestinaler Krisen (L oeper) hingewiesen. Wir müssen außerdem aufmerksam machen auf die Koexistenz und mehr noch auf die Aufeinanderfolge oder Alternation von Larynx-, Rektal- und seltener von Blasen-, Clitoris- und Lungenkrisen oder Krisen von Angina pectoris.

Fügen wir dem noch hinzu, daß man oft und $z u$ Recht die ziemlich häufige Koexistenz von Arthropathien und Magenerscheinungen konstatiert hat, so hätten wir die kurze Auseinandersetzung ihrer hauptsächlichsten Beziehungen zu dem Gesamtbild des klinischen Symptomenkomplexes der progressiven lokomotorischen Ataxie beendet.

\section{F. Diagnose.}

Die Diagnose der tabischen gastrischen Krise kann sich einem gewissermaßen auf den ersten Anhieb aufdrängen, wenn der Kranke schon ein vorgeschrittener Tabiker und der gastrische Symptomenkomplex sich in seiner typischen Form präsentiert. Aber es ist dem sicher nicht immer so. Eine „fruste" Krise läuft Gefahr, nicht erkannt zu werden und andererseits kann das Auftreten der Visceralgie in dem allerersten Stadium die genaue Deutung dieses frühzeitigen Symptoms der Sklerose der Hinterstränge sehr schwierig gestalten. Ohne hier alle sich überhaupt darbietenden Möglichkeiten zu erschöpfen, wollen wir hier nur in kurzen Zügen die Gedankengänge skizzieren, die sich einem logischerweise aufdrängen: die Erkenntnis, daß es sich tatsächlich um eine Magenkrise handelt; Ausschließung eines organischen Magenleidens; Nachweis der Beziehungen der Paroxysmen zu der Tabes.

a) Das erste dieser Diagnose, die in drei Abschnitte zerfällt, besteht darin, die gastrische Krise von den anderen schmerzhaften Symptomenkomplexen im Abdomen abzugrenzen, die sie vortäuschen können. Wir können hier nicht ausführlich auf alle Einzelheiten der Differentialdiagnose eingehen, die wir mit einigen Worten kurz abtun wollen.

Die oberflächlichen Abdominalneuralgien, die Neuralgien der letzten Interkostalnerven, die blitzartigen Schmerzen der Parie- 
talnetze dürfen mit der gastrischen Krise nicht verwechselt werden, mit der sie übrigens verbunden sein können. Der sehr oberflächliche Charakter der Schmerzen, das Vorhandensein von charakteristischen hyperästhetischen Punkten werden genügen, die Diagnose zu klären. Man darf nicht vergessen, daß ganz gewöhnliche Interkostalneuralgien mit Magenerscheinungen einhergehen, die zu Verwechslungen Anlaß geben (Loeper), aber die Analogien dieser Fälle mit der tabischen Gastralgie sind solche, $\mathrm{da} B$ der einzige Punkt, der unter solchen Umständen zu ent. scheiden wäre, der Zusammenhang oder Nichtzusammenhang des Symptomenkomplexes mit der Tabes ist. Der Sitz der Schmerzen bei der gastrischen Krise, die Empfindung ihrer Ausstrahlungen, die Natur der funktionellen Störungen (Magenintoleranz) werden die Diagnose gegenüber den Leber-, Nieren- und Darmkoliken, den Krisen bei Wandernieren, der Bleikolik, den abdominalen Krisen bei Purpura sichern, alles Symptomenkomplexe, die im übrigen eigenartige Charakterzüge bieten, die dem aufmerksamen Beobachter ihre richtige Deutung gestatten.

Die Perforationsperitonitis, namentlich diejenige im Anschluß an eine Magen- oder Duodenalperforation könnte höchstens einige Stunden zu Verwechslung Veranlassung geben. Es gibt aber ausnahmsweise Umstände, wo diese Differentialdiagnose ernste Schwierigkeiten bietet. Zum Beweise sei der Fall von gastrischer Krise bei einem Tabiker im Verlauf eines Abdominaltyphus angeführt, den Le Noir und Desbouis ganz kürzlich in den Bulletins de la Société nationale des hôpitaux de Paris berichtet haben.

Eine sehr ausgesprochene Ptose des Duodenum kann einen schmerzhaften Symptomenkomplex hervorrufen, der dem der gastrischen Krise ziemlich ähnelt. R. Leriche hat Gelegenheit gehabt, diese Läsion im Verlaufe eines operativen Eingriffes wegen der häufigen Wiederholung sehr schmerzhafter dyspeptischer Erscheinungen, die an Paroxysmen erinnerten, zu beobachten. Man begreift, daß unter solchen Umständen nur eine aufmerksame Röntgenuntersuchung gestatten würde, die beobachteten Erscheinungen auf ihre wahre Ursache zurückzuführen.

Manche Läsionen des Pankreas mit akutem Verlauf (akute Pankreatitis, hämorrhagische Pankreatitis) führen zu schmerz- 
haften Erscheinungen, von denen es sich schwer sagen läßt, ob man sie auf den Magen oder auf den Pankreas beziehen soll. Dasselbe „syndrome pancréatico-solaire" (Chaussard) ist manchmal mit paroxystischen Zügen im Verlaufe mancher chronischer Läsionen des Pankreas zu beobachten. Man muß diese Möglichkeiten kennen, um in manchen schwierigen Fällen nicht außer acht zu lassen, sie mit Hilfe einer methodischen Untersuchung auszuschalten.

Ebenso muß man die schmerzhaften Krisen kennen, die mit Veränderungen der Aorta und des sie umgebenden nervösen Plexus verbunden sind, Krisen, deren Symptomatologie nach den ursprünglichen Untersuchungen Potanis Prof. J. Teissier in ausgezeichneter Weise festgelegt hat.

Endlich wird die Annahme einer Vergiftung sich recht oft zu Beginn einer gastrischen Krise dem Kliniker aufdrängen, der sich durch eine schnelle Untersuchung und dahin zielende Fragen über das Vorliegen dieser Möglichkeit oder nicht zu versichern hat.

b) Der AusschluB eines organischen Magenleidens wird in der Regel leicht sein. Es ist sicher, daß das Ulcus und das Carcinom des Magens, das Ulcus duodeni manche Formen von Gastritis und besonders der Magensaftfluß mit schmerzhaften Paroxysmen einhergehen können, die auf den ersten Blick eine tabische Gastralgie vortäuschen können. In all diesen Fällen ermöglichen der Verlauf der Krise, ihr Anfangsmodus, die Kenntnis der Vorgeschichte, das oft weniger brüske Ende, das Fortbestehen schmerzhafter Erscheinungen oder zum mindesten funktioneller Störungen im Intervall der Krisen die Resultate der objektiven Untersuchung die Differentialdiagnose. Diese wird sich besonders schwierig gestalten gegenüber der intermittierenden Form des Magensaftflusses, die sich jedoch von ihr unterscheidet durch den oft weniger brüsken Beginn und dito Ende, durch eine weniger ausgesprochene Intoleranz des Magens, durch den sehr reichlichen Charakter des Erbrochenen, durch die häufige Hervorrufung von $Z$ wischenfällen durch einen Diätfehler, durch den beruhigenden Einfluß der alkalischen Medikation unu durch die Fortdauer der Hyperchlorhydrie im Intervall der Paroxysmen. Man darf jedoch nicht vergessen, daß die 
intermittierende Gastrosuccorrhöe den klinischen Ausdruck der tabischen Krise darstellen kann (Soupault).

Ebenso muB man sich daran erinnern, wie schwierig sich die Diagnose der Visceralgie gestaltet, wenn sie mit einem organischen Magenleiden vergesellschaftet auftritt. Kürzlich erst haben die Arbeiten von Duvernay, Klippel und Mathieu-Pierre Weil die Aufmerksamkeit auf die polyneuritischen Erscheinungen im Verlauf der Gastropathien und speziell des Ulcus ventriculi gelenkt. Dieser Umstand kann augenscheinlich eine Quelle der Verwechslung mit den gastrischen Krisen der Tabes darstellen.

c) Es bleibt uns endlich übrig nachzuweisen, daß der beobachtete visceralgische Paroxysmus tatsächlich mit der Tabes in $\mathrm{Zusammenhang}$ steht. Zu diesem Zwecke muß man alle anderen Ursachen, die sonst noch gastrische Krisen auslösen, ausschließen und vor allem nach anderweitigen Symptomen der Sklerose der Hinterstränge fahnden. Wir können diese hier nicht alle aufzählen. Wir haben im übrigen bereits oben auf die Beziehungen zwischen der gastrischen Krise und der allgemeinen Symptomatologie der Tabes hingewiesen. Wir habei dem bloß noch hinzuzufügen, daß man niemals verabsäumen darf, nach Syphilis in der Anamnese des Patienten zu fahnden, daß man im Bedarfsfalle die Wassermannsche Reaktion zu Hilfe nehmen muß und sich durch eine Lumbalpunktion von der Anoder Abwesenheit einer Lymphocytose des Liquor cerebrospinalis $\mathrm{zu}$ überzeugen hat.

Fehlt jedes Anzeichen von Tabes, selbst von „fruster", so suche man die Ursache der beobachteten Krise zu erforschen. Man muß sich dabei erinnern, daß sich unter ausnahmsweisen Umständen andere organische Erkrankungen des Zentralnervensystems durch ähnliche Erscheinungen äußern können, daß, allerdings seltener, Neurosen (Neurasthenie, Hysterie usw.) denselben Symptomenkomplex hervorrufen, daß eine große Zahl von Erkrankungen des Abdomens (Helminthiasis des Darmes, Appendicitis, Cholelithiasis, Ptosen usw.) ebenfalls imstande sind, das klinische Bild der gastrischen Krisen anzunehmen und da $B$ diese auch dyskrasischen Ursprungs (toxisch, autotoxisch, infektiös usw.) sein kann. Vom letzteren Standpunkt aus erwähnen 
wir, weil es sich um neue Forschungen handelt, daß Loeper mit Recht den Einfluß der Urikämie und Oxalämie betont hat. Ist man berechtigt, wenn jedes Symptom der Tabes fehlt und es unmöglich ist, die Ursache einer gastrischen Krise zu entdecken, die Diagnose auf eine essentielle gastrische Krise (De bove) oder, was auf dasselbe hinausläuft, auf das periodische Erbrechen v. Leydens zu stellen? Eine Reihe von Autoren (v. Leyden, Rémond, Mathieu, Debove u. a.) haben Fälle beobachtet, die uns dazu ein gewisses Recht geben. Man darf dabei aber nicht vergessen, daß die gastrischen Krisen lange vor jeder anderen Erscheinung der Tabes auftreten können. Daher hat Charcot mit Recht behaupten können, daß ,die Existenz der gastrischen Krisen, die denjenigen der Tabes in allem ähnlich sind und mit dieser nichts zu tun haben, keineswegs bewiesen ist". Auf jeden Fall stellten sie eine große Ausnahme dar.

II.

\section{Studium der Pathogenese.}

Die gastrische Magenkrise scheint a priori einem nervösen Mechanismus zugeschrieben werden zu müssen. Trotzdem ist es nicht ohne Interesse, den anatomischen $\mathrm{Zustand}$ des $\mathrm{Ma}$ gens zu betrachten, bevor wir in eine nähere Diskussion über die Pathogenese eintreten. Wir finden wertvolle Anhaltspunkte darüber in einer jüngst erschienenen Arbeit von Froment und Tolot ${ }^{1}$ ) und in der Dissertation eines ihrer Schüler, namens Riva ${ }^{2}$ ).

Die Autopsien von Tabikern mit gastrischen Krisen zeigen meist keine makroskopisch nachweisbare Läsion des Magens, jedoch ist es unmöglich festzustellen, mit welcher Häufigkeit dieses Organ tatsächlich intakt ist. Die Fälle, wo man nicht nur seine makroskopische, sondern auch mikroskopische Intaktheit konstatiert hat, sind äußerst selten. Eine einschlägige Beobachtung von Devic teilt Rivay in seiner Dissertation mit. Es ist übrigens berechtigt, hinzuzufügen, daß die Fälle von gastrischen Krisen, in denen eine histologische Untersuchung der Magen-

I) Lyon méd. I9o9.

2) Thèse de Lyon 1908-1909. 
schleimhaut vorgenommen wurde, keineswegs sehr zahlreich sind. Man findet im Gegenteil Magenläsionen in einer großen Anzahl von Tabes mit gastrischen Krisen. Die Art dieser Läsionen ist verschieden: Carcinom, Gastritis, allgemeine Linitis oder nur solche des Pylorus, Ulcerationen, Perigastritis, einfache Ekchymosen. Dieser organopathische Zustand des Magens kann von der Tabes ganz unabhängig sein und ein rein zufälliges Zusammentreffen darstellen. Dies gilt $z$. B. für das Carcinom, das übrigens selten ist, für die alkoholische und medikamentöse Gastritis. In einigen Fällen ist die Arteriosklerose des Magens mit im Spiel. Ein anderes Mal berechtigt das Aussehen der Läsionen dazu, sie wahrscheinlich von derselben Ursache abhängen zu lassen wie die Sklerose der Hinterstränge, d.h. von der Syphilis. Endlich muß man sich manchmal fragen, ob die am Magen beobachteten Veränderungen nicht direkt unter dem Einfluß des Nervensystems stehen. Eine derartige Frage ist namentlich hinsichtlich der Ekchymosen und Ulcerationen plausibel, die man nach Läsionen am Zentralnervensystem oder am Sympathikus und Vagus experimentell hat hervorbringen können.

Wenn wir die obigen Ausführungen zusammenfassen, so sehen wir, daß die Magenkrisen der Tabiker nicht einer einheitlichen Erklärung zugänglich sind. Wenn sie nicht selbst imstande sind, das Auftreten dieser Krisen zu erklären, so sind sie imstande, ihr Erscheinen zu begünstigen und ihr häufiges Wiederauftreten hervorzurufen. Außerdem geht die tabische Gastralgie unter solchen Umständen für gewöhnlich mit Magenblutungen einher.

Wir müssen nunmehr näher auf die Frage der Pathogenese eingehen. Die vorstehenden Ausführungen stellen gewissermaßen nur die Einleitung dar. Wir müssen nun fragen, welches die Nervenveränderungen sind, denen man die Magenerscheinungen im Verlaufe der Tabes zuschreiben muß.

Es ist ganz natürlich, daß man zuerst den Vagus beschuldigt hat, da man seine Rolle in der Leitung der sensiblen Eindrüicke vom Magen her als die wichtigste ansah. Seit I 880 schuldigt Buzzard, übrigens ohne genügende Beweise zu erbringen, die Sklerose des Vagus an. Seitdem haben verschiedene Autoren entweder über Läsionen des Ursprungskernes der sensiblen Fasern dieses Nerven oder über Veränderungen in seinem Stamm be- 
richtet. Wir weisen hin auf die Feststellungen von Pierret (Atrophie des bulbären Kernes des Vagus), Oppenheim, (Atrophie des Nervenstammes), Demange (Sklerose der Kerne des Vagus, Spinalis und der aufsteigenden Wurzel des Trigeminus), Kahler (diffuse Sklerose des Ependyms des IV. Ventrikels), Landouzy und Déjerine (Atrophie der Wurzeln und des Kerns des Spinalis und Vagus).

Die Mehrzahl der Autoren, die den 10. Hirnnerv für die Pathogenese der tabischen Gastralgie anschuldigen, schreiben dieser einen zentralen Lrsprung zu und nehmen mit Rosenthal namentlich einen anormalen Reizzustand desjenigen Teiles des sensiblen Vaguskernes an, in welchen die vom Magen her kommenden Sinneseindrücke endigen. Sie stützen sich dabei nicht nur auf anatomische Feststellungen, sondern auf die Kombination der Gastralgie mit einer Reihe von Erscheinungen, die man wohl mit Leichtigkeit auf einen bulbo-protuberantiellen Uisprung zurückführen kann: Spasmus des Larynx, Dyspnöe, Pulsverlangsamung, Angst, Schwindel, Bleichwerden des Gesichtes und Pupillendilatation.

Andere Autoren nehmen mit v. Le yden an, daß eine Läsion des Yerven selbst dazu hinreicht, um den beobachteten Symptomenkomplex zu schaffen, und $\mathrm{Cache}^{1}$ ) beschuldigt in seiner Dissertation sogar Läsionen des terminalen Vagus. J. Chalier und I. Nové-Josserand ${ }^{1}$ ) wollen ebenfalls den peripheren Veränderungen des Magenvagus und übrigens auch denen des Sympathikus cinen wichtigen Platz anweisen.

Allgemein gesprochen hat die Vagustheorie der tabischen gastrischen Krise eine Reihe von Argumenten gegen sich. Physiologische Arbeiten aus neuerer Zeit (M üller und Neumann) zielen darauf hin, die Bedeutung des Vagus für die sensible Leitung im Magen erheblich einzuschränken oder sogar in Zweifel $z u$ zithen. Der Nerv ist häufig intakt gefunden worden, dic Läsionen seines Kernes sind inkonstant und sogar sehr selten. Die sogenannten bulbären Symptome (Angst, Dyspnöc, Schwindel, Herzbeschwerden usw.) können der Übertragung der Reizung bis zum Bulbus und sogar bis zum Gehirn zugeschrieben werden.

I) Thèse de Paris 1897 . 
All diese Argumente haben Exner nicht verhindert, vor kurzem die doppelseitige subdiaphragmatische Vagotomie gegen die hartnäckigen gastrischen Krisen der Tabiker in Vorschlag $\mathrm{zu}$ bringen und auszuführen. In einem der beiden nach seiner Technik operierten Fälle findet er wichtige histologische Veränderungen der Vagi. Diese Veränderungen waren in dem zweiten Falle viel leichter. Diese Feststellungen sowie die bis jetzt von Exner erzielten Resultate, auf die wir weiter unten eingehen werden, sind nicht dazu geeignet, der Vagustheorie der gastrischen Krisen der Tabiker den ersten Platz zurückzuerobern.

Im Gegenteil ist die Mehrzahl der neueren Autoren geneigt, die hauptsächliche Rolle in der Pathogenese der tabischen Magenkrisen dem sympathischen System zuzuschreiben. Ihnen zufolge passiert die Mehrzahl der sensiblen Eindrücke vom Magen her kommend den Plexus solaris, das Ganglion semilunare, den großen Splanchnikus, erreicht dann auf dem Wege der Rami communicantes die hinteren Wurzeln des 6., 7., 8. und 9. Dorsalnerven (Head, Müller, Neumann, Laignel-Lavastine, Eppinger und $\mathrm{HeB}$ ).

Dic so interessanten Untersuchungen von $J$. C. Roux am Sympathikus der Tabiker haben gezeigt, daß in solchen Fällen die charakteristische Läsion dieses Systems das Zugrundegehen etwa der Hälfte der feinen myelinhaltigen Fasern mit Erhaltung der Ganglien ist. Die kleinen myelinhaltigen Fasern passieren aber die hinteren Wurzeln und sind vor allem sensible Leitungsfasern.

Es ist nach alledem berechtigt, der Läsion dieser Fasern und auch den Veränderungen des Systems der hinteren Wurzeln, zu denen sie in engen Beziehungen stehen, die visceralen Sensibilitätsstörungen im Verlaufe der Tabes und speziell die gastrischen Krisen zuzuschreiben.

Zugunsten dieser Auffassung lassen sich eine Reihe klinischer Argumente ins Feld führen: die so häufigen Sensationen des Beengtseins in der Thoraxgegend und die gürtelartig auftretenden Schmerzen, die man dabei oft beobachten kann, die Analgesie des epigastrischen oder solaren Punktes auf Druck, die man in der Regel sogar mitten während der Krise beobachten kann, ferner die Hyperästhesie der Haut der unteren Thorax- 
partie und der Haut des Epigastrium, die sich in einer Steigerung des epigastrischen Bauchdeckenreflexes äußert.

Diese oberflächliche Hyperästhesie ist nach $\mathrm{Head}$ der Ausdruck der Reizung des Rückenmarksegments, wo einerseits die Sympathikusäste, welche Reize gastrischen Ursprungs zuführen, andererseits die den peripheren Nerven angehörigen Äste, welche die sensible Innervation des Epigastrium und der unteren Thoraxpartie beherrschen. Das Leiden des Magens, oder allgemeiner ausgedrückt, die schmerzhafte Reizung seiner sensiblen Nerven, wäre durch einen Lokalisationsfehler auf die Peripherie reflektiert, in die Hautzone, deren sensible Fasern in demselben Rückenmarksegment endigen, zu erklären. Diese Hypothese $\mathrm{Heads}$, die viel Bestechendes für sich hat, ist von G.Guila in einer Kritik unterzogen, und die Resultate, die mehrere Nachuntersucher erzielt haben, haben sich nicht als so eindeutig erwiesen als diejenigen Heads. Warum soll man übrigens auf die Reizung des Rückenmarks zurückgreifen, um die Hyperästhesie der Haut im Verlaufe der gastrischen Krise bei Tabes zu erklären? Wäre diese nicht besser durch die Radikulalgie zu erklären? Es scheint plausibler, wenigstens hier die Wurzelläsionen anzuschuldigen, um die beobachteten Hyperästhesien zu ierklären. Die Anästhesien, die in derselben Hautzone im Moment der Krisen (Heitz) konstatiert worden sind, sind ebenfalls, scheint es, einer anderen Erklärung nicht zugänglich, abgesehen von den Anästhesien im Gefolge der einfachen Neuropathie, die übrigens nur ausnahmsweise zur Beobachtung kommt. Die Koexistenz eines Herpes im Gebiet der unteren Dorsalnerven, die in einigen Fällen von gastralgischen Krisen bei der Tabes (Siding, Leriche) beschrieben worden ist, ist ebenfalls ein Argument von hohem Wert zugunsten der Beteiligung der Radiculitis. Endlich dürfen wir nicht außer acht lassen, daß Loeper die Symptome von seiten des Verdauungstraktus, speziell die Gastralgien bei manchen radikulären Interkostalneuralgien, die im übrigen mit der Tabes nichts zu tun haben, in trefflicher Weise geschildert hat. Den von Loeper zusammengestellten einschlägigen Fällen schließt sich ein kürzlich von $\mathrm{P}$. Camus und $\mathrm{Bauffe}$ beobachteter Fall von großer Beweiskraft an.

Zusammengefaßt wäre also die gastrische Krise bei Tabes 
der Ausdruck einer Neuralgie oder einer Neuritis, die gleichzeitig vom Plexus coeliacus und den Wurzeln ausgeht. Die schmerzhaften Symptome, die sich dabei in erster Linie bemerkbar machen, können auf reflektorischem Wege das Auftreten anderer Symptome (Erbrechen, Singultus, Kontraktion der Bauchwand, Hypersekretion von Schleim und Magensaft usw.) bedingen, aber man darf nicht vergessen, daß manche Krisen unvollständig und daß besonders das schmerzhafte Element dabei vollständig fehlen kann. Ist es berechtigt, mit Exner die überwiegende Beteiligung des Vagus anzunehmen oder soll man sich vielmehr auf den Standpunkt stellen, daß die Läsionen im Bereich des Plexus coeliacus und der Wurzeln sich in manchen Fällen durch rein motorische, vasomotorische oder sekretorische Erscheinungen äußern können?

Man begreift leicht, daß Darmerscheinungen im Verlaufe der gastrischen Krisen in die Erscheinung treten können oder daß diese mit intestinalen oder Rektalkrisen alternieren können, im Hinblick auf die Tatsachen der physiologischen Synergie aller nervösen Apparate des Abdomens oder besser noch durch die Tatsache der Verteilung der Läsionen auf das sympathische System und das Ergriffensein einer Anzahl von Rückenmarkwurzeln hintereinander. Was das Auftreten von bulbären oder sogar cerebralen Symptomen betrifft, so muß es bei dieser Betrachtung der Dinge auf die Übertragung des Reizes auf die höheren Schichten des Zentralnervensystems bezogen werden.

Die logische Folgerung dieser selben Betrachtungsweise ist die Abhängigkeit mancher gastrischen Krisen bei Tabes von einer Beteiligung des hinteren radikulären Systems. Wir werden bald davon zu sprechen haben. Eine andere Folgerung dieser Betrachtung betrifft nicht mehr die Behandlung dieses Symptomenkomplexes, sondern dessen Prognose.

Trotz der vorhergehenden Ausführungen scheint es angebracht, der Vaguspathogenese der tabischen Gastralgie noch einen kleinen Platz einzuräumen und mit Förster Vaguskrisen neben Sympathikuskrisen anzunehmen. Wir werden auf die Unterscheidung dieser beiden Modalitäten bei der Besprechung der chirurgischen Behandlung des Symptomenkomplexes zurückkommen. 
Bevor wir diesen Abschnitt über die pathologische Physiologie schließen, müssen wir noch kurz auf die Bedeutung hinweisen, die $\mathrm{Pal}$ in Wien den Störungen des Blutdrucks bei der Produktion schmerzhafter abdominaler Krisen, speziell der tabischen Krisen beigemessen hat. Nach Pal sollen diese schmerzhaften Krisen in der Tat eine Funktion des erhöhten Blutdruckes, oder besser der Vasokonstruktion der Splanchnikusgefäße darstellen, deren erhöhter Blutdruck dafür den Beweis liefert. Es käme durch diesen Mechanismus ein anämischer Zustand der kleinen Arterienverzweigungen zustande und in den darunter liegenden Mesenterialarterien eine Rückstauung mit Dilation des Gefäßes und Ausdehnung des nervösen Plexus, das es umgibt. Diese vasokonstriktorischen Erscheinungen müssen den Läsionen des Systems der hinteren Wurzeln zugeschrieben werden mit dem gleichen Rechte wie die sekretorischen und vasomotorischen Erscheinungen, welche die Symptomatologie der tabischen Gastralgie bereichern können.

Daß die blutdrucksteigernden Medikamente wenigstens in einer gewissen Anzahl von Fällen eine günstige Wirkung auf den schmerzhaften Paroxysmus ausüben, kann nicht genügen, um die vorstehende Hypothese zu akzeptieren.

Man hat die Blutdrucksteigerung als sekundär zu dem Schmerze auffassen und beobachten können, daß sie um so ausgesprochener ist, je heftiger der Schmerz ist. Aber die Tatsache, daß der erhöhte Blutdruck bei unvollständigen Krisen vorkommen kann, führt dazu, die Blutdrucksteigerung und die gastrische Krise als zwei parallele Ereignisse aufzufassen. Die brüske Erhöhung des Blutdrucks ist vielleicht imstande, die schmerzhaften Erscheinungen $z u$ verschlimmern, ohne daß man ihr mit voller Sicherheit die pathogenetische Hauptrolle dabei zuschreiben darf (Curschmann, Hcitz und Noréro, Loeper).

III.

\section{Behandlung.}

Dic Therapeutik des tabischen Symptoms, das wir soeben miteinander studiert haben, war lange Zeit hindurch ausschließlich Besit\% der inneren Medizin. Sie bleibt auch heute noch als solche in Ehren bestehen. Jedoch scheinen uns die Hilfsmittel 
der modernen Chirurgen in der Behandlung der schweren rezidivierenden und hartnäckigen Formen der Visceralgie einer eingehenderen Besprechung wert. Dies ist um so interessanter, als es sich hierbei um ein ganz modernes Kapitel, das vielen Ärzten wohl noch nicht genügend bekannt ist, handelt.

\section{A. Interne Behandlung.}

Die Hilfsmittel der inneren Therapie sind zu geläufig, wenigstens was die Mehrzahl von ihnen betrifft, als daß es nötig wäre, uns länger über diesen Gegenstand zu verbreiten. Die Mannigfaltigkeit der in Vorschlag gebrachten Medikationen und Medikamente beweist an sich schon die Schwierigkeiten, die sich dem Arzte bei der Behandlung dieses Symptomes der Tabes entgegenstellen können. Wir werden zunächst die Behandlung erörtern, die im Verlaufe der Krise angezeigt sind, dann diejenigen im freien Intervall, um das Auftreten einer neuen ähnlichen Attacke zu verhüten.

Der gastralgische Paroxysmus hat zuerst die Unbeweglichkeit in der Rückenlage, dann die Aufhebung oder strenge Regelung der Diät zur Folge: Flüssige Diät, Eismilch oder einfach Eiswasser, mit oder ohne Zusatz von ein paar Tropfen Champagner oder Kaffee, Brauselimonade, Milch mit Zusatz von Laudanum (Vulpian). Jeder Tasse Milch werden 5-6 Tropfen von Laudanum Sydenhami hinzugesetzt.

Die Applikationen auf das Epigastrium, heiß oder kalt (Kataplasmen, wärmende Kompressen, Heißluftsack, Eisblase, pulverisierter Äther, Sprays), lindernde Salben (aus Chloroform, Opium, Hyoscyamin), Ableitung (Feuerspitzen, mit Morphium durchtränktes Blasenpflaster) werden mit einigem Nutzen angewandt.

Die sedativen Medikamente werden per os nur in den Fällen angewandt, wo die Intoleranz des Magens nicht eine solche ist, daß sie sich bei der geringsten Nahrungsaufnahme geltend macht. Man kann sich mit Opium oder dessen Alkaloiden behelfen, ferner mit Belladonna, Atropin, indischem Hanf, Kokain, Äther, Menthol, Chloroform- oder Bromoformwasser. Es ist leider nur sehr selten, daß diese sedativen Mittel eine schnelle und wohl ausgesprochene Linderung der Schmerzen herbeiführen.

In dem Falle einer absoluten gastrischen Intoleranz kann 
die rektale Anwendung von Opium, Belladonna oder Antipyrin gute Dienste leisten. Manchmal nützen auch Bromür- und Chloralklystiere.

Die Alkalien eignen sich bei den Formen mit Hyperchlorhydrie.

Es gibt jedoch viele Fälle, wo die Heftigkeit der Schmerzen Morphiuminjektionen erforderlich macht. Diese sollen nur selten wiederholt und rasch suspendiert werden, da der Patient wegen der Heftigkeit der Schmerzen dabei stark riskiert, sich dem Morphinismus zu ergeben.

Bei heftigen und länger anhaltenden Krisen versagen die oben genannten medizinischen Hilfsmittel und lassen kein anderes Hilfsmittel als wiederholte Morphiuminjektionen übrig. Man muß in diesem Falle sein möglichstes tun, um die Dauer dieser qualvollen Zustände nach Kräften abzukürzen und vor allem alles daran setzen, die Heftigkeit der Paroxysmen zu lindern.

Die Anwendung von Santonin (drei Oblaten à o, Io g pro die) ist geeignet, die Erfüllung dieser Wünsche zu unterstützen. Man kann auch nach den Indikationen von $\mathrm{Pal}$ blutdruckherabsetzende Mittel (Inhalationen von Amylnitrit oder subkutane Injektionen, täglich I-2, bis 4 mal, von I proz. salpetersauren Natron) geben. Der Vorzug ist namentlich den Inhalationen zu geben, weil sie imstande sind, eine sofortige Linderung der Schmerzen herbeizuführen, die in einigen Fällen (Heitz und Noréro) von Dauer sein kann.

Das Ablassen einer größeren Menge von Liquor cerebrospinalis vermittelst Lumbalpunktion (Debove, Babinski, Castaigne) hat einigen Patienten gute Dienste leisten können. Es kann sich daran eine subarachnoidale Injektion von Kokain, Stovain oder Novocain anschließen, die in den Händen mehrerer Autoren befriedigende Resultate ergeben hat. Der eine von uns hat zweimal unter ähnlichen Umständen die Lumbalpunktion ohne günstige Wirkung ausgeführt. Es bestand keine Drucksteigerung des Liquor, und dieser selbst bot bei der cytologischen Untersuchung nichts Pathologisches. Ganz kürzlich haben G. Mouriquand und Cot te einen Fall von tabischen gastrischen Krisen publiziert, in dem das Ablassen von $25 \mathrm{ccm}$ Liquor cerebrospinalis von bedrohlichen Synkopeerscheinungen gefolgt war. 
Wir machen ferner noch auf die einfache Ableitung auf die Wirbelsäule aufmerksam (Bespritzen mit Äthylchlorid oder Feuerspitzen). Was die Anwendung der Röntgenstrahlen anbelangt, so sind sie nur sclten angewandt worden. Die Galvanisation ist trotz ihrer cinfachen praktischen Ausführbarkcit ebenfalls nur sehr selten angewandt worden. Eine lange Elektrode (positiver Pol) wird auf das Epigastrium aufgesetzt, die andere auf die Lendenoder Brustwirbel, und man läßt einen Strom von 20 oder 30 Milliampère ca. Io Minuten lang laufen.

Selbstverständlich ist, daß, wenn es der Allgemeinzustand des Kranken erlaubt, man unverzüglich Strychnin-, Koffein-, Ätheroder Kampferölinjektionen macht. Das einfache oder mit Kaffein versetzte künstliche Serum, das ebenfalls subkutan zu injizieren ist, darf ebenfalls nicht außer acht gelassen werden, ebenso die Klistiere von künstlichem Serum und die Nährklistiere.

Die Komplikationen, wie z. B. die im Verlaufe einer Krise auftretenden Blutungen, sind ebenfalls die Quelle spezieller Indikationen.

Dic Prophylaxe eines so dramatisch anmutenden Symptomenkomplexes, wie es die Krise der tabischen Gastralgie ist, liegt sehr nahe. Hier ist in erster Linie der Einfluß einer guten Nahrungshygiene zu nennen, ferner die Vermeidung von Alkohol und die Unterdrückung oder zum mindesten in jedem Falle die Regelung der gewöhnlichen medikamentösen Injektionen.

Was die Behandlung der Tabes selbst betrifft, so darf diese niemals außer acht gelassen werden. Aber leider nur allzu häufig bietet sie keinen großen präventiven Schutz gegen die Rückkehr der Paroxysmen. Diese erlegen in jedem Falle eine weise Mäßigung in dem Gebrauch der Quecksilbermedikation auf, die lediglich in Form von intramuskulären Injektionen anzuwenden ist. Man hat noch kürzlich die intraarchanoidalen Injektionen von Elektromercurol nach den praktischen Erfahrungen des Herrn Professor Carrieu gerüht. Man wird ferner nicht die Feuerspitzen längs der Wirbelsäule außer acht lassen. Die Anwendung von salpetrigsaurem Natron per os oder subkutan, die R a y mond warm empfohlen hat, wird nur sehr selten ausgeführt. 
B. Chirurgische Behandlung (Dr. Leriche).

I. Indikationen.

Mißlingt die interne Behandlung, so kann man mit den uns heute zur Verfügung stehenden Mitteln besser und erfolgreicher als bisher verhindern, dal3 ein Tabiker mit gastrischen Krisen in kürzester Zeit zum chronischen Morphinisten wird. Handelt es sich um eine hartnäckige oder rezidivierende Krise, läßt die Intensität der Schmerzen dem Unglücklichen keine Ruhe noch Rast, bewirken die absolute Unmöglichkeit, Nahrung zu sich zu nehmen und die außergewöhnliche IIäufigkeit von Erbrechen eine schnelle Abmagerung, die das Leben gefährden kann, so soll man versuchen, durch einen zweckmäßigen Eingriff die visceralen Beschwerden zu lindern. Indem man dies tut, kann man vielleicht jede Wiederkehr derselben für die Zukunft verhindern.

Kurzum, es soll nach dem heutigen Stand der Dinge ein 'Tabiker mit gastrischen Krisen operiert werden.

\section{Historisches.}

Der Gedanke, chirurgisch zu intervenieren, um einer durch ihre Heftigkeit und Dauer gefährlichen Krise erfolgreich zu begegnen, ist übrigens neuesten Datums. Zuerst hat man sich mehr ,par hasard" darin versucht, indem man auf den tabischen Magen die chirurgische Panacee, soweit sie in der Magenpathologie Mode war, anwandte, d.h. je nach den Jahren die digitale Dehnung des Pylorus und dann die Gastroenterostomie. Diese rein empirischen Versuche, die pathogenetisch nicht im mindesten begründet waren, konnten kein Resultat liefern und haben es auch tatsächlich nicht getan. Abgesehen von den von $\mathrm{Dubar}$ allein (1898) oder in (iemeinschaft mit I.e roy veröffentlichten Fällen hat der direkte Eingriff am Magen nur Mißerfolge aufzuweisen. Man hat sie nicht immer publiziert. Jedoch haben in den letzten Jahren König, Küttner und ich selbst eine Reihe derartiger Beobachtungen mitgeteilt, deren es noch viel mehr gibt.

In Wirklichkeit konnte sich eine zweckmäßige Art des Angriffes der gastrischen Krise nur auf einem sorgfältigen Studium der pathologischen Physiologic aufbauen. 
Um die verschiedenen Etappen der Frage richtig zu verstehen, ist es notwendig, sich das Schema der Mageninnervation genau ins Gedächtnis zurückzurufen.

Der Magen erhält sensible Fasern vom Vagus, der zugleich sein motorischer Nerv ist, und vom Sympathikus, der sein hauptsächlichster sekretorischer Nerv ist.

Die sympathischen Äste besitzen einen komplizierten Durchgang, um schließlich das Dorsalmark zu erreichen, wo sich ihre Zentren befinden. Sie konzentrieren sich zu allererst in der Gegend des Ganglion semilunare. Von diesem Ganglion aus gelangen sie in die beiden Nervi splanchnici. Der Splanchnicus major steigt in den Thorax hinauf und entsendet seine konstituierenden Fasern in die Reihe der Rami communicantes, die zu den 9., 8., 7., 6. und 5. Dorsalnerven hinziehen. Man nimmt an, daß dieser Nerv das Gros der sympathischen Fasern des Magens darstellt. Der Splanchnicus minor entsendet nach dem Dorsalmark die Rami communicantes der I2., II. und 10. Dorsalnerven. Man meint, daß er in sich hauptsächlich intestinale Fasern vereinigt.

Zwischen diesen beiden Systemen existieren in der Plexusgegend zahlreiche Anastomosen. Ebenso gibt es deren mit den beiden ersten Lumbalnerven.

Es besteht zwischen all den genannten Nerven eine Art pathologische Solidarität.

Auf diesem langen Wege vom Magen bis zum Rückenmark ist der Sympathikus, dessen Läsion die Magenkrise bedingt, chirurgisch an mehreren Stellen erreichbar, in der Gegend des Plexus solaris, in der Gegend der Rami communicantes und des Ganglion spinale durch Vermittlung des Nervus intercostalis und endlich im Gebiete der hinteren Wurzel. Der Nervus splanchnicus interthoracicus ist tief verborgen im Mediastinum und daher schwer. zugänglich.

Nach diesen Vorbemerkungen gehen wir auf die verschiedenen chirurgischen Verfahren bei den gastrischen Krisen ein.

Im Jahre I 900 stellte Jaboulay in der Dissertation seines Schülers Termier die Hypothese auf, daß bei gastrischen Krisen vielleicht eine Dehnung des Plexus solaris gerechtfertigt wäre. 
Im Jahre I 906 teilten Vallas und Cotte ein günstiges Resultat in einem Falle mit, in dem die Besserung zwei Jahre anhielt.

Dies war gewissermaßen das Vorspiel zu den methodischen und präzisen Arbeiten Försters.

Im Jahre I909 schlug dieser vor, bei gastrischen Krisen die Operation auszuführen, die seinen Namen trägt, und die darin besteht, die intradurale Reaktion der 7., 8., 9. und Io. hinteren Wurzeln auszuführen. Er meinte, daß man auf diese Weise den zentripetalen Weg der Magensensibilität unterbrechen und infolgedessen die Krise in dem Augenblick kupieren könnte, in dem sie auf reflektorischem Wege zu entstehen im Begriffe ist. Die Tatsachen gaben ihm recht. Er ließ durch $\mathrm{K} \ddot{u} \mathrm{ttn}$ er einen ersten Patienten operieren, und ziemlich rasch griff man von den verschiedensten Seiten seinen Vorschlag auf. Auf dem 37. deutschen Chirurgenkongre $ß$ im Jahre I9lo zeigte eine Diskussion über die Förstersche Operation deren gute Resultate. Im Anschluß an einen selbst beobachteten und operierten Fall stellte ich im folgenden Jahre in Gemeinschaft mit Cotte in einem kritischen Artikel ${ }^{1}$ ) I I einschlägige Fälle zusammen, welche die Bilanz der Durchschneidung der hinteren Wurzeln bei der Tabes darstellten. Ende r9I zählte Förster 28 Fälle, von denen 23 befriedigende Resultate geliefert hatten und 2 mit einem vollständigen Mißerfolg endigten. Bis zum I. IX. I912 habe ich 62 Fälle zusammenstellen können, die sich in der Dissertation meines Schülers $\mathrm{Mazade}^{2}$ ) finden. Die Totalität dieser Fälle setzt die Richtigkeit der operativen Anschauungen, die von nun $a b$ über alle Diskussion erhaben ist, ins rechte Licht. Jedoch war die ursprünglich von Förster ersonnene Operation von einer wirklichen Schwierigkeit, da sie hauptsächlich an geschwächten und morphinistischen Kranken zur Ausführung kam.

Um ihre Prognose zu verbessern, gab Guleke im Jahre I 9 Io eine wichtige technische Modifikation an, die darin besteht, daß man die Rhizotomie nicht mehr wie früher im Inneren des duralen

I) Journ. de chirurgie I9IJ.

2) Thèse de Lyon 1912. In dieser Arbeit sind 42 Fälle zusammengestellt. Seitdem haben wir zwei weitere Fälle mit gutem operativem Resultat. 
Überzuges, sondern im Spatium epidurale ausführt. Auf diese Weise vermeidet man das Abfließen von Liquor cerebrospinalis und dies vereinfacht den Eingriff und seine Folgen. Es stellt somit einen tatsächlichen Fortschritt dar.

Franke geht auf andere Weise vor. Bei einem Patienten, der die Vornahme der Försterschen Operation verweigert hatte, hatte er die Idee, den sensiblen Weg auf indirektem Wege dadurch zu erreichen, daß er die den Wurzeln, die er hätte durchschneiden sollen, entsprechenden Intercostalnerven extrahierte. Sein Patient genas und bekam keine Krisen wieder. Er teilte dies Resultat auf dem 39. Deutschen Chirurgenkongre $B$ mit anläßlich der Diskussion, die wir erwähnt haben; und seitdem haben einige Autoren, darunter auch ich, den Wert dieses Verfahrens, das sich auf dem Försterschen aufbaut und ihm gegenüber den Vorzug der größeren Einfachheit und leichteren Zugänglichkeit hat, hervorgehoben. Wir wollen gleich jetzt vorwegnehmen, daß es unserer Ansicht nach in manchen Fällen das Verfahren der Wahl darstellt.

All die genannten operativen Methoden zielen darauf hin, den Sympathikus als den hauptsächlichsten Weg der Magensensibilität zu erreichen. Manche Fälle geben hingegen zu denken, daß der Vagus manchmal bei den Magenkrisen mit im Spiel ist. Im Hinblick auf diese Fälle hat Exner ganz kürzlich die Durchschneidung der beiden Vagi in der Gegend der Cardia in Vorschlag gebracht und ausgeführt. Diese Sektion führt zu atonischer Lähmung des Magens. Das Verfahren ist wenig verlockend a priori, läßt sich aber doch rechtfertigen.

Das wären die chirurgischen Methoden, die wir zu studieren haben, bevor wir, wenn möglich, präzisieren, was wir angesichts einer hartnäckigen Magenkrise zu tun haben. Aber das ist noch nicht alles.

Ganz kürzlich hat sich $\mathrm{K}$ önig gefragt, ob es nicht möglich wäre, die Vorteile der blutigen Behandlung ohne operativen Eingriff zu erhalten, ob man nicht alle chirurgische definitive Durchschneidung der Wurzeln durch ihre zeitliche Aufhebung ersetzen könne, indem man eine Lösung von Novocain-Adrenalin in die Gegend der Austrittsstelle der Dorsalnerven in jiziert.

In der Tat injizierte König bei einem Tabiker, der seit 
mehreren Jahren an heftigen Magenkrisen litt und dessen $\mathrm{Zu}$ stand sich durch eine Gastroenterostomie nicht besserte, im Verlaufe einer sehr heftigen Krise $100 \mathrm{ccm}$ einer 0,05 proz. Novocainlösung tief in die Rückenmuskulatur an der Austrittsstelle der Wurzeln zwischen dem 6. und Io. Dorsalwirbel. Der Erfolg trat auf der Stelle ein, eine Wiederkehr der Krisen wurde auf dieselbe Art und mit demselben Resultat behandelt. Es gibt also ein einfaches Mittel, das beachtet zu werden verdient.

III. Vergleichendes Studium der verschiedenen Methoden.

a) Eingriffe am Magen.

Man hat die digitale Zerrung des Pylorus und die Gastroenterostomie ausgeführt. Wenn man sich an die sporadisch mitgeteilten Beobachtungen hält, würde man ihre Anwendung vielleicht für gerechtfertigt erachten. Dies ist jedoch tatsächlich nicht der Fall. Einigen erfolgreichen Fällen, bei denen wir die Dauer der Besserung überdies nicht kennen, könnten wir eine ganze Reihe vollständiger Mißerfolge entgegenstellen. Die Mißerfolge haben nichts Erstaunliches. Die direkte Operation am tabischen Magen kann durch nichts gerechtfertigt werden. Sie könnte nur in dem einen Falle verteidigt werden, wenn organische viscerale Läsionen mit der Tabes vergesellschaftet auftreten. Andererseits kann man nach dem, was man heutzutage über die Funktion des Magens nach der Gastroenterostomie wei $\beta$, bei den Tabikern, die keine Pylorusstenose haben, das rasche Unbrauchbarwerden der künstlichen Öffnung und infolgedessen die Unwirksamkeit dieses Verfahrens voraussehen.

b) Eingriff an den hinteren Wurzeln. Förstersche Operation.

a) Das Prinzip.

Die leitende Idee Försters war folgende: Die Analyse der Krise lehrt, daß sie sich aus drei wesentlichen Elementen zusammensetzt : motorischen Störungen (Erbrechen, Singultus, Kontraktion der Bauchmuskulatur), sekretorischen Störungen (Hypersekretion des Magensaftes, vermehrte Schleimabsonderung) und sensiblen Störungen. Letztere scheinen zeitlich die ersten zu 
also sekundäre auffassen, im AnschluB an die Reizung der Magenschleimhaut.

Wenn das erste Symptom der Krise eine sensible Störung: ist, so besteht das therapeutische Problem darin, zu wissen, welches die Nerven sind, deren Veränderung die Hyperästhesie des Magens bewirken. Im normalen Zustand ziehen die sensiblen Äste des Magens zum Teil zum Sympathikus, zum Teil zum Vagus hin. Erstere gehen von der Magenschleimhaut zum Plexus solaris. Nachdem sie die Ganglia semilunaria und den Splanchnikus passiert haben, endigen sie in die hinteren Wurzeln aus, indem sie hauptsächlich den Durchgang der Rami communicantes des 7., 8., 9. und Io. Dorsalnerven benutzen. Nun lehrt eine Anzahl klinischer Beobachtungen, daß bei der Tabes namentlich diese Fasern mit im Spiel sind. Die gastrische Krise ist der Ausdruck einer Radiculitis wie die große Mehrzahl der tabischen Krisen. Es ist daher nichts Auffälliges, daß der Prozeß die sympathischen Fasern in Mitleidenschaft zieht, die den Wurzeln zugeführt werden.

Kurzum: es sind bei den visceralen Tabesformen hauptsächlich die sympathischen Äste, die erkrankt scheinen. Daher die Anschauung Försters, daß man, indem man die Sensibilität der Magenschleimhaut aufhebt, diese Äste in ihrem radikulären Durchtritt durchschneidet und damit die Krisen lindert.

B) Die Technik.

Um diese Durchschneidung der Wurzeln auszuführen, hat man heutzutage zwei Verfahren: das von Förster und das von Guleke.

Das Verfahren von Förster besteht hauptsächlich darin, den Duralsack in der Medianlinie nach einer zweckentsprechenden Laminektomie zu eröffnen und die 5., 6., 7., 8., 9. und 10. hintere Dorsalwurzeln in der Gegend ihres Eintrittes in das Rückenmark zu resezieren nach Eröffnung der Rückenmarkshüllen in der Medianlinie. Es gibt zu einem sofortigen großen Verlust von Liquor cerebrospinalis und $z u$ der späteren Bildung einer Ansammlung dieser Flüssigkeit zwischen den Meningen und den Muskeln Veranlassung. Diese encystierte Ansammlung wächst in den ersten Tagen unaufhörlich, ist schmerzhaft und stört die operativen Folgen. Daher gaben wir bis in die letzte Zeit dem 
Verfahren von Guleke den Vorzug, das wir an der Leiche studiert haben und in einem Falle von Parkinsonscher Krankheit ${ }^{1}$ ) zur Anwendung brachten. Dies Verfahren hat zum Gegenstand, die Wurzeln zu resezieren, ohne den Duralsack in der Medianlinie $z u$ eröffnen. $Z u$ diesem Zweck klappt man den Duraüberzug erst nach der einen, dann nach der anderen Seite zurück. Dieser Zug läßt keine Tubus erscheinen, die eine Art Etui der vorderen und hinteren Wurzel darstellen, die sich an jener Stelle und, nachdem je für sich seine eigne Hülle erreicht haben, in einer gemeinsamen Hülle eingeschlossen verschmelzen. Dort reseziert man die hinteren Wurzeln, nachdem man sie sorgfältig getrennt hat ${ }^{2}$ ). Das Verfahren ist weniger schwierig als das Förstersche. Es scheint ihm daher vorgezogen werden zu müssen. Leider ist das $\mathrm{Gulekesche} \mathrm{Verfahren} \mathrm{nicht} \mathrm{immer}$ zu verwenden, manchmal ist es sehr schwer auszuführen. Daher geht unsere jetzige Meinung dahin, man solle es nach Möglichkeit anzuwenden versuchen. Stellt es sich aber als zu schwierig heraus, so soll man nicht zögern, die Meningen zu eröffnen.

r) Die Resultate.

Die Förstersche Operation erfordert Zeit, sorgfältige Ausführung, sie ist schwierig und bedeutet darum stets einen schweren Eingriff. Immerhin ist das Verfahren nicht so ernst zu bewerten, wie dies manche gutheißen. Die Mortalität beträgt höchstens 12-I 5 Proz. In Zukunft wird diese Mortalitätsziffer sicher noch weiter heruntergehen. Kurzum man kann der Operation im Prinzip ihre große Gefahr nicht mehr mit Recht einwenden ${ }^{3}$ ).

I) Über chirurgischen Eingriff bei Parkinsonscher Krankheit. Neurolog. Zentralbl. 1912.

2) In zwei neuerdings operierten Fällen von tabischen Krisen haben wir das Gulekesche Verfahren zur Ausführung gebracht. In dem einen haben wir im Augenblick der Operation selbst kein Abfließen von Liquor cerebrospinalis bei den Durchschneidungen der hinteren Wurzel beobachtet. Jedoch trat in den nächsten Tagen eine Ansammlung ein, deren langsame Filtrierung die operativen Folgen etwas beeinträchtigt. In dem anderen Falle ging die Durchschneidung jeder Wurzeln mit dem Austritt von etwas Liquor einher, es fand jedoch kein sekundärer postoperativer Abfluß von Liquor statt.

3) Wir haben bis jetzt fünfmal die Förstersche Operation ausgeführt, ohne einen Todesfall erlebt zu haben. 
Vom therapeutischen Standpunkt a us hat man im allgemeinen sehr gute unmittelbare Resultate. Sogleich nach der Operation oder in zunehmendem Maße in den folgenden Tagen hören die Krisen auf, verschwinden die Schmerzen. Die Nahrungsaufnahme wird wieder möglich, der Kranke kommt wieder zu Kräften und nimmt an Gewicht zu. Es bleiben jedoch ziemlich häufig schmerzhafte Erscheinungen im Bereich des Darmtraktus bestehen, aber vom gastrischen Standpunkt ist der Zweck vollauf erreicht.

Ist dieses Resultat dauerhaft? Ja, meistens. Ein Patient $\mathrm{K}$ üttners ist seit I 5 Monaten vollkommen geheilt. Ein Fall von v. Angerer befindet sich $\mathrm{I}^{1 / 2} \mathrm{Jahr}$ nach der Operation in ausgezeichnetem Zustande und hat $24 \mathrm{~kg}$ zugenommen. Ein ebenfalls von v. Angerer operierter Patient klagt seit einem Jahr nicht mehr über Magenschmerzen. Kurzum, es gibt eine erkleckliche Zahl von Heilungen, die in etwa zwei Drittel der Fälle nach der Operation eintreten.

Aber außer diesen mehr oder weniger vollständigen Erfolgen kommen auch Rezidive vor, die ebenso schwer und schmerzhaft sind wie die ursprünglichen Symptome der Krankheit, und dies nach einer scheinbaren Heilung von mehreren Monaten. Diese Rezidive sind übrigens leicht zu erklären und können vielleicht in Zukunft vermieden werden. In fast allen Fällen mit Rezidiv sieht man nämlich, daß der Chirurg sich darauf beschränkt hat, drei Wurzeln jederseits $z u$ resezieren. $D$ as ist vollständig ungenügend. Man muB mindestens fünf Wurzeln jederseits durchschneiden und vorzugsweise sechs oder sieben in den Fällen, wo das Allgemeinbefinden des Patienten dies gestattet. Sehr oft treten nämlich Magenkrisen mit Darmkrisen vergesellschaftet auf. Die einen werden von den anderen beeinflußt. Der Magen reagiert in unangenehmer Weise auf den Darm; daher kommt es zu Rezidiven. Es ist wahrscheinlich zur Sicherung des Resultats notwendig, wie Förster jetzt meint, Wurzeldurchschneidungen vorzunehmen, die von der fünften Dorsalwurzel nach oben bis zur zwölften Dorsalwurzel nach unten gehen. Man kann diesen ausgedehnten Eingriff nicht immer einzeitig ausführen, er ist jedoch in einer Anzahl von Fällen das Ziel, das man sich zu stecken hat. 
Mit neuen Hilfsmitteln soll man daher in Zukunft an die Behandlung der Rezidive herantreten.

Es verbleibt uns noch die Frage der vollständigen Mißerfolge. Sie sind nicht sehr zahlreich, sie kommen aber vor. Nach unserer Statistik zählen wir ihrer sechs. Man kann sich in diesen Fällen fragen, ob es sich hier nicht um Krisen gehandelt hat, die zum Vagus ausgingen. Wir haben oben gesehen, daß der Nervus vagus manchmal in der Pathogenese gewisser Krisen angeschuldigt wurde und erinnern an das bereits von uns Gesagte. Es gibt bei der Tabes zwei Arten von gastrischen Krisen: die Sympathikuskrise, die den gewöhnlichen Typus darstellt und die Vaguskrise, die charakterisiert ist durch Steigerung des epigastrischen Reflexes und durch das gleichzeitige Bestehen von Larynxstörungen und Herzerscheinungen. Diese Fälle fallen nicht unter den Indikationsbereich der Försterschen Operation. Es ist möglich, daß die mitgeteilten Mißerfolge den Beweis dafür liefern.

Kurzum, die Förstersche Operation ist ein a usgezeichnetes Mittel zur Beeinflussung hartnäckiger Krisen. Sie liefert manchmal wunderbare Resultate, und meist, etwa in zweiDrittel derFälle, große Besserungen. Sie stellt jedoch einen ernsten und schwierigen Eingriff dar, dessen Indikation wohl erwogen sein muß und der unter gewissen Umstän. den kontraindiziert ist.

\section{Frankesche Operation.}

a) Das Prinzip.

Sie besteht, wie oben ausgeführt, darin, daß man sehr langsam nach der Thierschschen Methode das zentrale Ende der Interkostalnerven extrahiert, die den Wurzeln entsprechen, welche den Magensympathikus in Aktion setzen. Die leitende Idee Frankes war die, daß man wahrscheinlich, ,auf diese Weise Ganglienfasern extrahieren könnte, die eine wichtige Rolle in dem Reflexbogen spielen", und auf diese Weise die sensible Bahn unterbricht.

In der Tat kann man sich an der Leiche leicht von der Richtigkeit dieser Voraussetzung überzeugen. Wenn man, wie 
wir das in Gemeinschaft mit Cotte getan haben, den ganzen Tunnel, den der Nervus intercostalis hinein in die Gewebe und die Knochenruine des Ganglion verfolgt, herausheben läßt, nachdem das Rückenmark freigelegt ist, so sieht man, daß die an den Interkostalnerven ausgeführten Züge hauptsächlich auf die Ganglien wirken und daß sie sich nur in schwachem Maße der sensiblen Wurzel selbst bei ihrem Eintritt in das Mark mitteilen.

Am Lebenden ist es so: Franke hat in seinem ersten Falle zweimal ein Ganglienfragment herausgefördert. Maire und Par turier haben ähnliche Beobachtungen gemacht, ebenso Cotte und Mouriquand in einem ganz kürzlich operierten Falle. In unserem Falle dagegen haben wir nichts dergleichen beobachtet.

Kurzum: die Extraktion des Interkostalnerven wirkt mit Sicherheit und in aktiver Weise auf das Spinalganglion und die Rami communicantes. Sie kann auf diese Weise die Übertragung sensibler gastrischer Reize in enormem Maße verändern.

Förster hat dagegen eingewandt, daß man vielleicht Gefahr läuft, die motorischen Wurzeln zu extrahieren oder ernste Läsionen des Rückenmarkes hervorzurufen oder endlich Zerreißungen von radikulären Arteriolen zu setzen, deren Läsion vielleicht eine Querschnittsmyelitis bedingen könnte. Es scheint uns, daß diese Einwände einer Kritik nicht standhalten. Sie werden angesichts der beobachteten Fälle einfach gegenstandslos.

ß) Die Technik.

Technisch ist die Frankesche Operation sehr leicht. Durch einen langen vertikalen Einschnitt an jeder Seite der Wirbelsäule, vier Querfinger von der Dornfortsatzlinie oder weiter außerhalb (was nach unserer jetzigen Erfahrung besser ist), erhält man den 6., 7., 8., 9. und I0. Interkostalnerven. Dieses bietet keine Schwierigkeit. Der Nerv wird auf einem Haken sehr langsam und fortwährend ausgezogen, bis er nachgibt. Dies soll nach Franke für jeden Nerven 3-4 Minuten erfordern. Vielleicht ist es besser nach den Untersuchungen van Gehuchtens eine brutale Extraktion vorzunehmen, um die Chamatolyse der Ganglien sicherer hervorzurufen.

Die einzige Gefahr der Operation besteht darin, daß sie sich in der Nähe der Pleura abspielt, die leicht bei der Auf- 
suchung der Nerven verletzt werden kann. Wegen dieser Gefahr ziehen wir es vor, etwas weiter von der Medianlinie zu incidieren, wie wir früher angegeben haben. Der Intercostalis internus ist mehr entwickelt und bildet ein wirksames Hindernis.

Y) Die Resultate.

Die Resultate der Frankeschen Operation sind ausgezeichnete. Wir kennen bisher ro Fälle. In allen, mit Ausnahme von einem, trat die operative Heilung ohne $Z$ wischenfall ein. Eine der Patienten Frankes starb allerdings an eitriger Pleuritis nach einigen Wochen. Es scheint uns, daß derartige Zwischenfälle sich in Zukunft vermeiden lassen.

Vom therapeutischen Standpunkt aus hat die Extraktion der Interkostalnerven neben bemerkenswerten Resultaten zwei Mißerfolge gezeitigt. Diese beiden Mißerfolge sind von Clairmont berichtet worden, der in der Sauerbruchschen Kammer (man fragt sich warum) den 5.-9. Interkostalnerv durchschnitt und extrahierte. Die Krisen hörten zwar augenblicks auf, um jedoch nach 4-6 Wochen wieder aufzutreten. Man kann diese beiden Mißerfolge auf analoge Weise wie die bei der Försterschen Operation damit erklären, daß sich der Eingriff auf eine zu beschränkte Anzahl von Nerven bezog. Es ist jedoch unnötig, weitere Worte über diese beiden Fälle zu verlieren, die von Clairmont ganz kurz mitgeteilt worden sind. Im übrigen hat die Frankesche Operation ausgezeichnete Resultate geliefert. Eine Patientin, deren Krankengeschichte wir mitgeteilt haben, litt an heftigen Magenbeschwerden: sie war abgemagert, kachektisch und vertrug dabei anstandslos die Extraktion von fünf Nerven beiderseits. Die Krisen verschwanden. Bald wurde die normale Ernährung wieder aufgenommen und zwei Monate später hatte die Kranke I I kg an Gewicht zugenommen. Der Erfolg hält bis jetzt drei Monate an ${ }^{1}$ ).

Ebenso hat der erste von Franke operierte Patient seit mehr als zwei Jahren kein Rezidiv mehr gehabt. Man kann

1) Seitdem hat diese Kranke ein Rezidiv nach dem intestinalen Typus bekommen. Wir haben, um ihre Beschwerden zu lindern, den 4., II. und I2. Intercostalnerven und die beiden $N$. genito abdominales beiderseits extrahiert (vgl. Presse médicale, 27. März 1912 und Lyon médical, I9. Mai 1912). Seitdem, d. h. seit drei Monaten, geht es der Patientin ausgezeichnet. 
daher sagen, daß die Frankesche Operation einen ausgezeichneten, sehr wirksamen und sehr nützlichen Eingriff darstellt. Sie ist einfach und leicht aus$z$ uf ühren, nicht gefährlich und verdient daher in einer Anzahl von Fällen den Vorzug vor der Försterschen Operation, die sicherlich schwieriger und in ihren Folgen bedenklicher ist.

Soll man damit zugleich aussprechen, daß man die Frankesche Operation in allen Fällen als die Operation der Wahl ansehen müsse. Ich glaube nicht. Man kann ihr zwei Einwände machen: erstens ist sie weniger präzis als die Förstersche Operation und andrerseits hat sie den schweren Nachteil, daß sie gemischte Nerven durchtrennt. Man beobachtet nach Ausführung der Operation fast immer eine sehr deutliche Parese der Bauchwand in der subumbilikalen Region. Diese Parese kann die Verhältnisse einer echten Eventration annehmen, die für den Patienten äußerst lästig ist. Aus diesem Grunde würden wir zögern (und wir haben gezögert), diese Operation bei denjenigen Patienten, denen noch ein langes aktives Leben, bevorsteht, auszuführen.

D. Eingriff am Plexus solaris.

a) Das Prinzip.

Wir haben daran erinnert, daß Jaboulay früher die Dehnung des Plexus solaris bei der Behandlung mancher hartnäckigen Visceralgien warm befürwortet hatte, da der Sympathikus und infolgedessen der Plexus solaris zum Teil die sensible Innervation des Abdomens versorgt. Die zentripetalen Nerven des Magens passieren den Plexus solaris. Infolgedessen ist ein Eingriff in dieser Gegend bei gastrischen Krisen vollauf berechtigt. Jaboulay hat in der Dissertation seines Schülers Termier ${ }^{1}$ ) eine übrigens ziemlich versteckte darauf bezügliche Anspielung gemacht, die allen Autoren entgangen ist.

B) Die Technik.

Sie besteht im wesentlichen darin, den Plexus durch das kleine Netz und die hintere Höhle in der Gegend der Aorta und des Plexus coeliacus aufzusuchen.

I) Thèse de Lyon 19 Io. 
Y) Die Resultate.

Man verfügt bisher über zu wenig Fälle, um dic Methode zu beurteilen. In dem von Cotte veröffentlichten Falle von Vallas wurde der während der Krise operierte Patient vollständig von seinen Beschwerden geheilt. Zwei Jahre lang traten bei ihm keinerlei Beschwerden auf, dann kam es wieder zu einem Rezidiv.

In einem von uns selbst beobachteten Fall haben wir keinerlei Resultat erzielt. Das Urteil über die Jaboula ysche Methode muß3 also so lange offen bleiben, bis neue Fälle dazu beigebracht werden. Wir nehmen uns vor, sie bei der ersten sich darbietenden Gelegenheit zu studieren.

E. Die doppelseitige Vagotomie.

(a) Das Prinzip.

Die Physiologie lehrt, daß der Vagus einer der sensiblen Nerven des Magens ist. Die pathologischen Anatomen haben, wie oben erwähnt, manchmal Läsionen dieses Nerven bei ' $\mathrm{Ta}$ bikern mit Magenkrisen beobachtet. Endlich hat die klinische Analyse Förster dazu geführt, zwei Typen von Krisen zu unterscheiden, auf dic wir bereits hingewiesen haben: die Sympathikus- und die Vaguskrise.

Endlich ist der Vagus der Nerv, der das Erbrechen auslöst.

Man ersieht daraus, daß es logisch war, den Vagus in geeigneten Fällen anzugreifen. Das hat Exner versucht.

B) Die Technik.

Exner hat in den beiden einzigen 1 ) bisher bekannt gewordenen Fällen von doppelseitiger Vagusdurchschneidung die beiden Vagi in der Kardiagegend durchschnitten. Dies bietet keine großen Schwierigkeiten, jedoch schließt sich unmittelbar an diese Operation eine atonische Lähmung des Magens, Hypersekretion und reflektorische Kontraktion des Pylorus an. Dabei ist es nötig, die Entleerung des Magens zu sichern. Daher machte Exner im Anschluß an seine beiden Fingriffe eine Gastrotomie und

I) Seit der Fertigstellung dieses Aufsatzes haben v. F.isclsberg über drei Fälle von Vagusdurchschneidungen (davon zwei mit Frfolg) und Küttner über einen Mißerfolg berichtet. 
legte einen Drain durch den Pyloraus bis zum Duodenum gehend an.

r) Die Resultate.

Von den beiden von Exner operierten Patienten wurde einer geheilt, der andere starb. Bei diesem letzteren kombinierte sich nach einigen Tagen tatsächlicher Besserung (es handelte sich um äußerst schmerzhafte und besonders hartnäckige Krisen bei einem Patienten, der sich wegen seiner Leiden das Leben zu nehmen versucht hatte) eine Magenatonie mit einer beträchtlichen Dilatation des Magens. Die Gastrotomie wurde inkontinent und der Tod trat nach drei Wochen infolge Fortschreiten der Schwäche ein.

In dem andern Falle dagegen war das Resultat vortrefflich. Nach Ablauf von drei Wochen verminderte sich die Atonie, der Pylorus-Duodenaldrain wurde weggelassen und die Fistel schloß sich. Drei Monate später ging es dem Patienten gut, die Krisen waren bei ihm nicht wieder aufgetreten.

Zusammengefaßt ist also zu sagen, daß sich über diese theore. tisch sehr berechtigte Operation noch kein abschließendes Urteil fällen läßt, weil noch $\mathrm{zu}$ wenig einschlägige Beobachtungen vorliegen. Es scheint, als wenn sie wirklich, wenn auch ausnahmsweise indiziert ist, nämlich bei anormalen gastrischen Krisen, bei denen die klinische Untersuchung ergibt, daß es sich um Vaguskrisen handelt.

\section{Schlußbemerkungen.}

Zum Schluß erhebt sich die Frage:

Welche von all den genannten Methoden soll man wählen und wie soll man vorgehen, wenn die chirurgische Indikation sich bei einem Tabiker erhebt?

Das erste, was zu tun ist, ist die Natur der Krise zu erkennen versuchen und ihre Hauptelemente zu unterscheiden.

Handelt es sich um eine sehr schmerzhafte Krise mit starkem Erbrechen, Tachykardie, Aussetzen des Pulses und Larynxkrisen, so wird man eine Vaguskrise befürchten müssen. Man muß den Beweis dazu erbringen suchen und kann dann daran denken, die doppelseitige Vagotomie nach Exner auszuführen. Dieser Fall wird jedoch eine Ausnahme darstellen. 
SO CADE u. LEkiche, Über die gastrischen Krisen bei der Tabes dorsalis.

Für gewöhnlich jedoch dürfte es sich um Sympathikuskrisen handeln. Man muß dann vom einfachen zum komplizierten Verfahren vorgehen. Man soll mit dem gutartigsten Eingriff beginnen und sich die großen operativen Verfahren als ultimum refugium aufsparen.

Das einfachste und gutartigste Verfahren besteht darin, zuerst die Königsche Methode anzuwenden, d. h. in die Rückenmuskeln, beiderseits von der Dornfortsatzlinie 1 oo $\mathrm{ccm}$ einer 0,5 proz. Novokainlösung zu injizieren. Um eine dauernde Wirkung zu erzielen, könnte man vielleicht das Novokain durch Alkohol ersetzen und die gastrische Krise nach Art einer Facialisneuralgie behandeln.

Sollte dies Verfahren mißlingen, so wird man sich der Frankeschen Operation zu bedienen haben: man wird den 5.-7. Interkostalnerven extrahieren (die Zahl wäre festzustellen nach dem Ergebnis der kombinierten Magendarmerscheinungen). Man könnte ferner die Jaboula ysche Operation, Dehnung des Plexus solaris, versuchen.

Sollte auch diese Methode versagen oder sollte es zu Rezidiven kommen, so wird man seine Zuflucht zu der Försterschen Operation nach dem von Guleke angegebenen epiduralen Verfahren nehmen. Man wird, wenn möglich, sieben Wurzeln beiderseits durchtrennen.

Trotzdem glauben wir, daß man bei den noch widerstandsfähigen Kranken sofort die Wurzeldurchschneidung vornehmen kann, wenn es sich um sehr schwere Krisen mit deutlicher Anteilnahme des Darmes handelt.

Es wäre sehr überraschend, wenn es mit Hilfe eines derartigen Verfahrens nicht gelänge, dem unglücklichen Patienten eine wirkliche Linderung seiner furchtbaren Beschwerden zu verschaffen.

Es ist nicht mehr wie recht und billig, das Verdienst F ö rster zuzuschreiben, dessen geniale Anschauungen in so glücklicher Weise ein gutes Stück der Neurologie umgewälzt haben. 